\title{
CÁLCULO DE VISCOSIDADE DE LÍQUIDOS COM A EDE CUBIC- PLUS-ASSOCIATION INCORPORADA AO MODELO EYRING
}

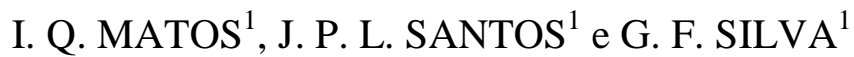 \\ ${ }^{1}$ Universidade Federal de Sergipe, Departamento de Engenharia Química \\ E-mail para contato: josepaixaosantos@yahoo.com.br
}

\begin{abstract}
RESUMO - A viscosidade é uma propriedade de transporte fundamental para o desenvolvimento de processos nas indústrias petroquímica, petrolífera, química e de outras indústrias que envolvem o transporte de fluidos. Cálculos de viscosidade são baseados no princípio dos estados correspondentes ou na teoria de Eyring. Assim, o objetivo do presente trabalho é incorporar a equação de estado Cubic-Plusassociation (EdE CPA) na teoria de Eyring para correlacionar e predizer a viscosidade dos componentes puros que formam ligações de hidrogênio metanol, etanol e 2-propanol e das misturas metanol+etanol, metanol+2-propanol e etanol+ 2propanol. A EdE CPA forneceu resultados satisfatórios tanto para componentes puros quanto para misturas binários.
\end{abstract}

\section{INTRODUÇÃO}

A viscosidade é uma propriedade de transporte fundamental para projetar equipamentos nas indústrias química, petroquímica e petróleo nas quais estão relacionadas ao transporte, agitação, filtração e troca de calor de fluidos. Apesar disso, não há uma teoria satisfatória para propriedades de transporte de gases densos e líquidos reais. As dificuldades inerentes ao estudo dessas propriedades são atribuídas a medidas experimentais pouco confiáveis e a complexidade no tratamento teórico. Por exemplo, correlações para viscosidade são empíricas ou têm uma sólida base teórica. Os modelos mais usados estão baseados na teoria desenvolvida por Eyring que representa uma alternativa aos modelos baseados na mecânica estatística e às correlações empíricas. A teoria de Eyring é baseada na energia livre de ativação em excesso de escoamento e esta é relacionada com modelos de energia livre em excesso e, consequentemente, com coeficientes de fugacidade obtidos através de uma equação de estado (Hildwein e Stephan, 1992). Para correlacionar a viscosidade e a densidade, é necessário associar uma equação de estado para calcular a energia livre de Gibbs em excesso e, no caso desse trabalho, será usada a equação de estado Cubic Plus Association ( EdE CPA) (Kontogeorgis et al., 1996). Esta é uma equação híbrida que possui termos provenientes de diferentes teorias, ou seja, um termo provém da equação SRK (Soave-Redlich-Kwong), para contabilizar as interações físicas, e o outro provém da equação SAFT (Statistical-Associating-Fluid-Theory), para contabilizar a formação de ligações de hidrogênio. A EdE CPA vem fornecendo resultados bastante exitosos para a modelagem de sistemas que possuem ligações de hidrogênio, como os que envolvem alcoóis. Além disso, seu custo computacional é mais alto do que as das uma EdE's cúbicas, mas é mais 
baixo do que o custo de EdE's baseadas puramente na mecânica estatística como, por exemplo, a EdE SAFT e suas variantes. Sendo assim, esse trabalho tem como objetivo calcular a viscosidade dos componentes puros metanol, etanol e 2-propanol e calcular a viscosidade e densidade molar das misturas binárias metanol + etanol, metanol + 2-propanol e etanol + 2-propanol.

\section{MODELAGEM TERMODINÂMICA}

Para os componentes puros, o modelo para viscosidade usado (Xuan et al., 2006) está relacionado com o volume molar e a pressão pela seguinte equação:

$$
\mu=k_{1} \exp \left(\frac{P V^{\ddagger}}{R T}\right)
$$

onde, $k_{1}$ é um parâmetro pré-fator e $k_{2}$ é um coeficiente de escala que relaciona o volume de ativação, $V^{\neq}$, com o volume molar $\left(V^{\neq}=k_{2} V_{m}\right)$. A viscosidade de misturas é estimada a partir da seguinte relação (Lin, 2007):

$$
(\mu V)_{m}=(\mu V)_{I D} \exp \left(\frac{\sigma G^{e x}}{R T}\right)
$$

Onde $(\mu V)_{I D}$ refere-se a solução ideal e pode ser relacionada as propriedades e composição dos componentes puros pela equação abaixo.

$$
\ln (\mu V)_{I D}=\sum_{i=1}^{n_{c}} x_{i} \ln \left(\mu_{i} V_{i}\right)
$$

O modelo de energia de Gibbs em excesso, $G^{e x}$, é calculado através da relação entre os coeficientes de fugacidade do componente na mistura $\phi_{i}$ e o coeficiente de fugacidade do componente puro $\phi_{i}^{0}$.

$$
G^{e x}=R T \sum_{i=1}^{n_{c}} x_{i}\left(\ln \phi_{i}-\ln \phi_{i}^{0}\right)
$$

O parâmetro $\sigma$ da Equação (1) é considerado como dependente da composição do componente 1 da mistura para aumentar a precisão da estimação e está relação é definida como:

$$
\sigma=\sigma_{0}+x_{1} \sigma_{1}
$$

Como já foi dito anteriormente, é necessário uma equação para os cálculos das propriedades termodinâmicas necessárias para obter a viscosidade. A equação de estado usada é 
a EdE CPA cuja forma funcional está descrita a seguir (Michelsen, 2006):

$$
P^{C P A}=\frac{R T}{V_{m}-b}-\frac{a}{V_{m}\left(V_{m}+b\right)}+\frac{1}{2}\left(\frac{R T}{\bar{V}}\right)\left(1+\frac{1}{\bar{V}} \frac{\partial \ln g}{\partial(1 / \bar{V})}\right) \sum_{i} x_{i} \sum_{A_{i}}\left(1-X_{A_{i}}\right)
$$

A descrição dos termos e parâmetros desta EdE pode ser vista em outros trabalhos (Michelsen, 2006). Os parâmetros $k_{1}$ e $k_{2}$ são estimados a partir da minimização da função objetivo (Equação 7) empregando dados de viscosidade de componentes puros. Já os parâmetros de mistura $\sigma_{0}, \sigma_{1}$ e $k_{i j}$ são estimados a partir da minimização da função objetivo (Equação 8) empregando dados de viscosidade e densidade de mistura. O método de otimização utilizado foi método estocástico de enxame de partículas (Schwaab et al., 2008).

$$
\begin{aligned}
& F O=\sum_{i=1}^{N}\left[\frac{\left|\mu^{c a l}{ }_{i}-\mu^{\exp }{ }_{i}\right|}{\mu^{\exp }{ }_{i}}\right]^{2} \\
& F O=\sum_{i=1}^{N}\left[\frac{\left|\mu^{c a l}{ }_{i}-\mu^{\exp }{ }_{i}\right|}{\mu^{\exp }{ }_{i}}\right]^{2}+\sum_{i=1}^{N}\left[\frac{\left|\rho_{i}^{c a l}-\rho_{i}^{\exp }\right|}{\rho^{\exp }{ }_{i}}\right]^{2}
\end{aligned}
$$

Neste trabalho, os parâmetros característicos do componente puro da EdE CPA foram estimados a partir de dados de pressão de vapor e densidade molar com o esquema de associação de 2B (Huang e Radosz, 1990).

\section{RESULTADOS E DISCUSSÕES}

Os parâmetros da EdE CPA estimados a partir de dados de pressão de vapor e densidade de componente puro (DIADEM, 2000) fornecem baixos desvios* em relação aos dados experimentais, como pode ser visto na Tabela 1 , o que os qualificam para uma boa predição da viscosidade experimental. Os parâmetros $k_{1}$ e $k_{2}$ estimados a partir de dados experimentais de viscosidade (Tanaka et al., 1987) estão expostos na Tabela 2. Tais parâmetros resultaram em baixos desvios, comprovando a acurácia da EdE CPA em correlacionar viscosidade de compostoos altamente polares.

Tabela 1 - Parâmetros da EdE CPA

\begin{tabular}{|l|c|c|l|c|c|l|l|}
\hline compostos & $\mathrm{a}_{0}\left(\mathrm{bar} \cdot \mathrm{L}^{2} \cdot \mathrm{mol}^{-2}\right)$ & $\mathrm{b}(\mathrm{L} / \mathrm{mol})$ & $\mathrm{c}_{1}$ & $\varepsilon^{\mathrm{AB}}\left(\mathrm{bar} \cdot \mathrm{L} \cdot \mathrm{mol}^{-1}\right)$ & $\beta^{\mathrm{AB}} \cdot 10^{3}$ & $\Delta \rho^{*}$ & $\Delta \mathrm{P}$ \\
\hline Etanol & 7,49 & 0,049 & 0,72 & 231,2 & 9,4 & 0,3 & 0,7 \\
\hline Metanol & 3,77 & 0,031 & 0,83 & 213,4 & 42,2 & 1,0 & 0,7 \\
\hline 2-propanol & 11,26 & 0,065 & 0,99 & 197,8 & 9,2 & 0,6 & 0,4 \\
\hline
\end{tabular}

* Todos os desvios relativos das propriedades foram calculados pela seguinte equação $\Delta Z=100 / N \sum_{i=1}^{N}\left[\left|Z^{c a l}{ }_{i}-Z^{\exp }{ }_{i}\right| / Z^{\exp }{ }_{i}\right]$ 
Tabela 2 - Parâmetros de viscosidade componente puro

\begin{tabular}{|l|c|c|c|}
\hline compostos & $k_{1}$ & $k_{2}$ & $\Delta \mu$ \\
\hline Etanol & 1,11 & 0,248 & 0,8 \\
\hline Metanol & 0,56 & 0,292 & 0,9 \\
\hline 2-propanol & 2,07 & 0,279 & 0,8 \\
\hline
\end{tabular}

Para efeito de comparação, o estudo das misturas binárias foi feito empregando duas estratégias: a primeira utiliza somente o parâmetro de interação $k_{i j}$, assumindo $\sigma$ igual a 1,0 e a segunda leva em consideração três parâmetros $k_{i j}, \sigma_{0}$ e $\sigma_{1}$ para representar as propriedades em estudo. Os respectivos parâmetros para ambas as estratégias podem ser vistos na Tabela 3. Os valores elevados dos parâmetros $\sigma_{1}$ para as misturas indicam uma alta dependência do coeficiente de correção da energia livre de Gibbs em excesso em relação ao componente 1 da mistura binária.

Tabela 3 - Resultados para misturas binárias com três e um parâmetro

\begin{tabular}{|l|l|l|l|l|l|l|l|l|}
\hline \multicolumn{1}{|c|}{ Mistura } & $k_{i j}$ & $\sigma_{0}$ & $\sigma_{1}$ & $\Delta \rho$ & $\Delta \mu$ & $k_{i j}$ & $\Delta \rho$ & $\Delta \mu$ \\
\hline Metanol+Etanol & $-0,0026$ & 1,594 & $-9,19$ & 0,5 & 0,1 & 0,022 & 0,3 & 0,8 \\
\hline Metanol+2-propanol & $-0,0102$ & 0,004 & $-25,11$ & 0,4 & 0,3 & 0,104 & 1,0 & 4,4 \\
\hline Etanol+2-propanol & $-0,0003$ & 2,887 & $-18,18$ & 0,2 & 0,1 & 0,014 & 0,3 & 1,6 \\
\hline
\end{tabular}

As correlações de densidade molar das misturas metanol + etanol, metanol + 2-propanol e etanol+2-propanol podem ser vistas nas Figuras 1 a 3. Os resultados encontrados para ambas as estratégias foram satisfatórios e similares. A única mistura em que houve grande discrepância entre as estratégias foi a mistura metanol+2-propanol. Este comportamento pode ser atribuído a forte dependência da predição da densidade molar da mistura com a composição.

O uso de três parâmetros melhoram consideravelmente as correlações da viscosidade para as misturas estudadas, como mostra as Figuras 4 a 6 . Pode-se observar que para a mistura metanol + 2- propanol, a estratégia com apenas o parâmetro $k_{i j}$ não consegue correlacionar com os dados experimentais, enquanto que a estratégia com o parâmetro dependente da composição apresenta resultado bastante satisfatório. Para as misturas metanol + etanol e etanol + 2-propanol o modelo com apenas um parâmetro apresentou grandes desvios nas composições extremas do componente um. Os resultados mostram que o uso de um parâmetro dependente da composição aumenta significativamente a acurácia dos resultados para a predição da viscosidade.

\section{CONCLUSÕES}

Desenvolver modelos capazes de predizer propriedades de transporte como a viscosidade é essencial para a indústria e o modelo de Eyring com a EdE CPA acoplada forneceu bons resultados para as misturas estudadas. Diferentemente de outros trabalhos que empregaram equações de estados que não contabilizavam implicitamente ligações de hidrogênio, aqui a EdE utilizada contem um termo que descreve fisicamente a formação de ligações de hidrogênio. 
Logo, poucos parâmetros foram necessários para representar o comportamento de viscosidade de mistura. Além disso, o emprego do parâmetro $\sigma$ dependente da composição aumentou a acurácia do modelo.

\section{REFERÊNCIAS}

American Institute of Chemical Engineers, Design Institute for Physical Properties, DIADEM, 2000.

HILDWEIN, H.; STEPHAN, K. Equation of state and equations for viscosity and thermal conductivity of non-polar and polar pure fluids based on the significant-liquid-structure theory. Chemical Enginering Science, v. 48, p. 2005-2023, 1993.

HUANG, S. H.; RADOSZ, M. equation of state for small, large, polydisperse, and associating molecules. Ind. Eng. Chem. Res., p. 2284-2294, 1990.

KONTOGEORGIS, G. M.; VOUTSAS, E. C.; YAKOUMIS, I. V.; TASSIOS, D. P. An equation of state for associating fluids. Ind. Eng. Chem. Res., v. 35, p. 4310-4318, 1996.

LIN, C. F.; HSIEH, H. M.; LIANG-SUN, L. Estimations of the viscosities of binary mixtures with different equations of state and mixing rules. J. Chin. Inst. Chem. Engrs., v. 38, p. 1-19, 2007.

MICHELSEN, M. L. Robust and efficient solution procedures for association models, Ind. Eng. Chem. Res., v. 45, p. 8449-8453, 2006.

SCHWAAB, M.; BISCAIA E.C.; MONTEIRO, J.L.; PINTO, J.C. Nonlinear parameter estimation through particle swarm optimization. Chem. Eng. Sc., v.63, n. 6, p. 1542-1552, 2008.

TANAKA, Y; MATSUDA, Y.; FUJIWARA, H.; KUBOTA, H.; MAKITA, T. Viscosity of (water + alcohol) mixtures under high pressure. Int. J. Thermophys, v. 8, p. 147-163, 1987.

WEI, I. C.; ROWLEY, R. L. Binary liquid mixture viscosities and densities. J. Chem. Eng. Data, v. 29, p. 332-335, 1984.

XUAN, A.; WU, Y.; PENG, C.; MA, P. Correlation of the viscosity of pure liquids at high pressures based on an equation of state. Fluid Phase Equilib., v. 240, p. 15-21, 2006. 


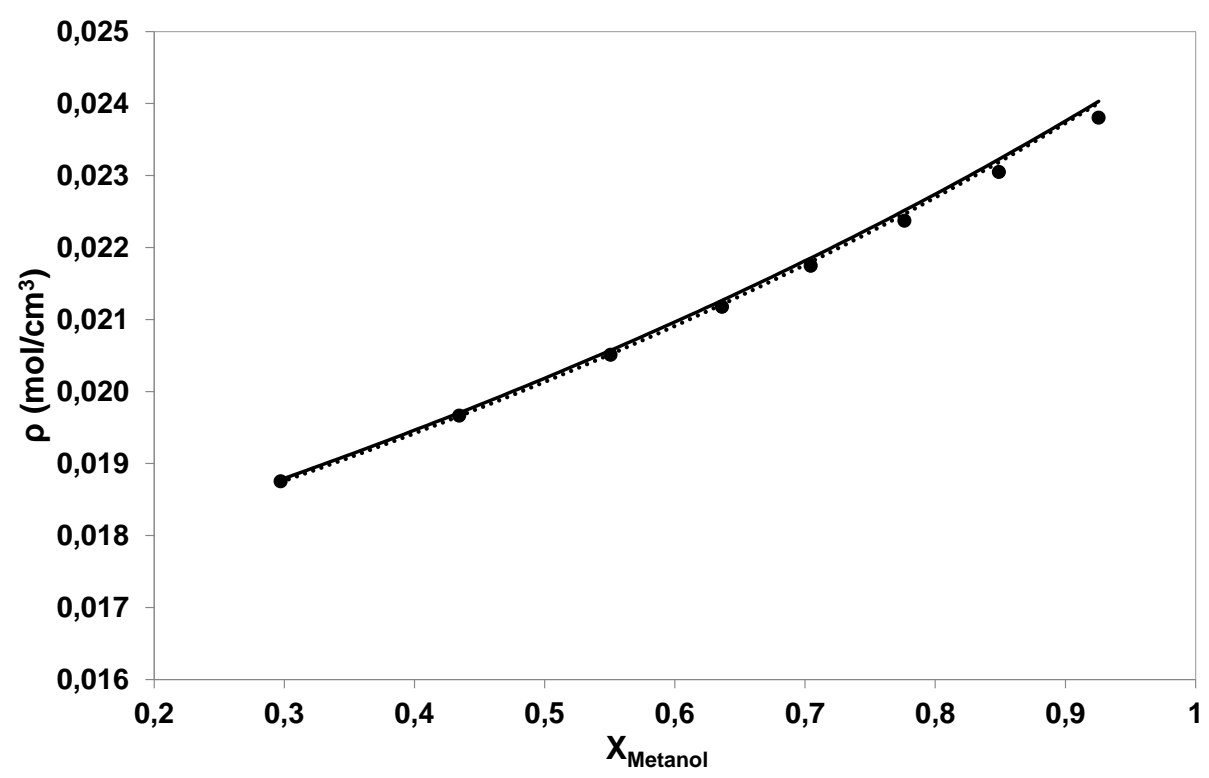

Figura 1 - Densidade da mistura metanol + etanol. $k_{i j}, \sigma_{0}, \sigma_{1}(-)$ e $k_{i j}(\cdots) .(\bullet)$ (Wei e Rowley, 1984).

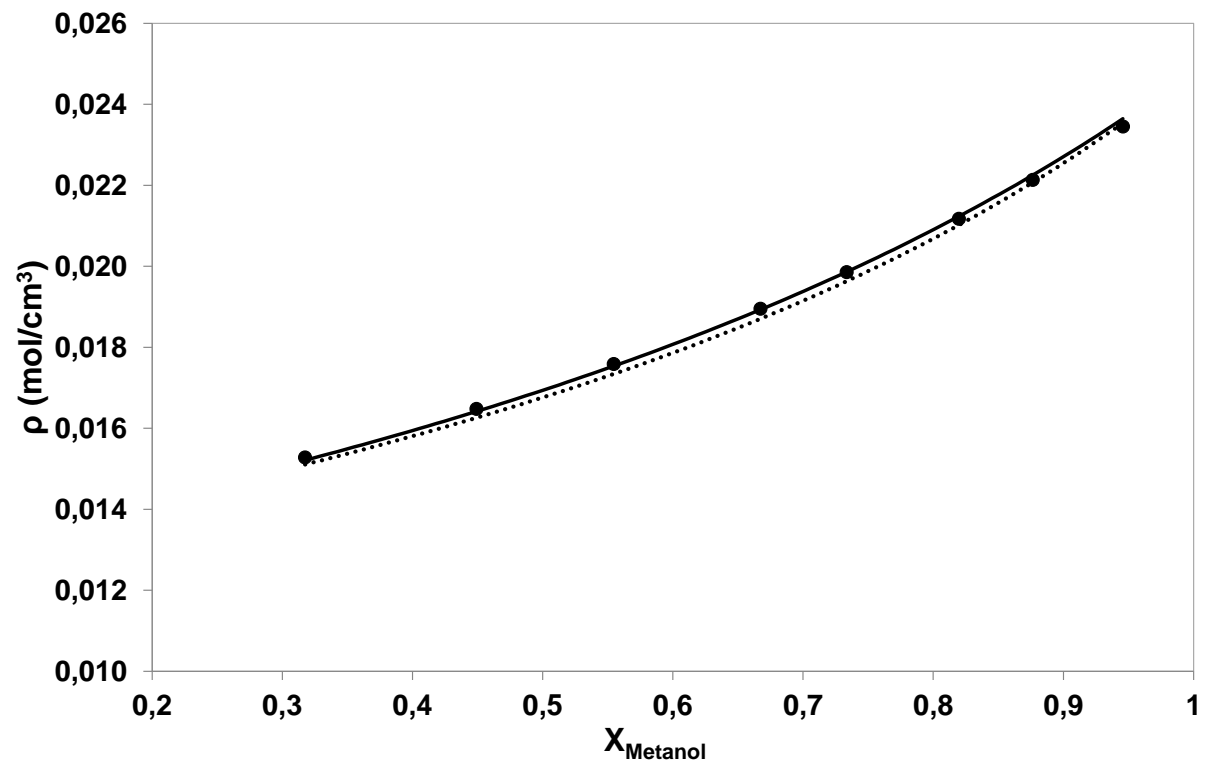

Figura 2 - Densidade da mistura metanol +2- propanol. $k_{i j}, \sigma_{0}, \sigma_{1}(-)$ e $k_{i j}(\cdots)$. (•) (Wei e Rowley, 1984). 


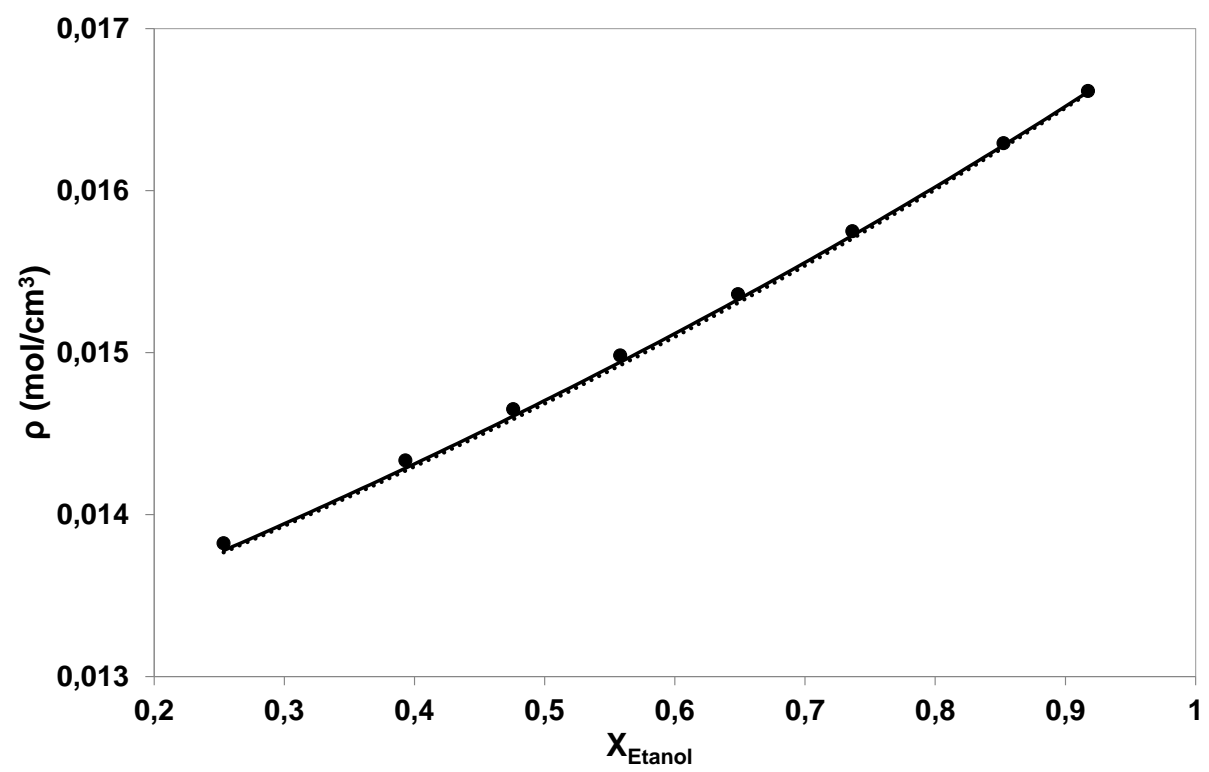

Figura 3 - Densidade da mistura etanol +2- propanol. $k_{i j}, \sigma_{0}, \sigma_{1}(-)$ e $k_{i j}(\cdots) .(\bullet)$ (Wei e Rowley, 1984)

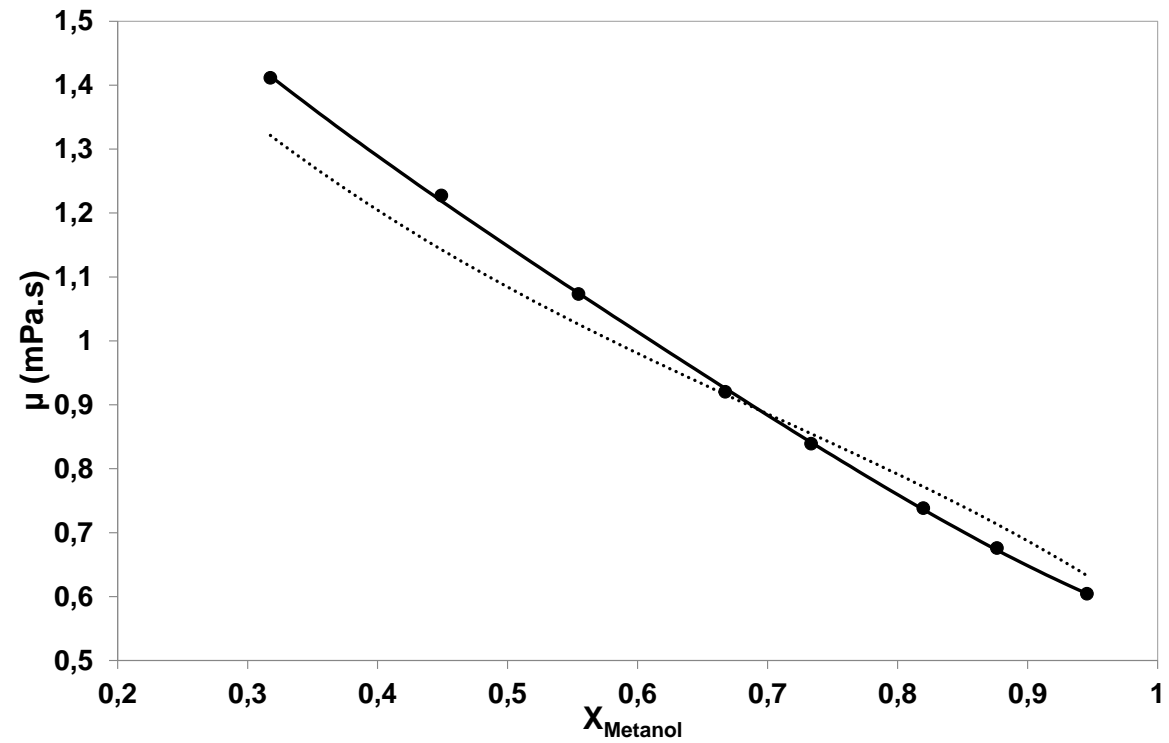

Figura 4 - viscosidade da mistura metanol +2- propanol. $k_{i j}, \sigma_{0}, \sigma_{1}(-)$ e $k_{i j}(\cdots)$. (•) (Wei e Rowley, 1984). 


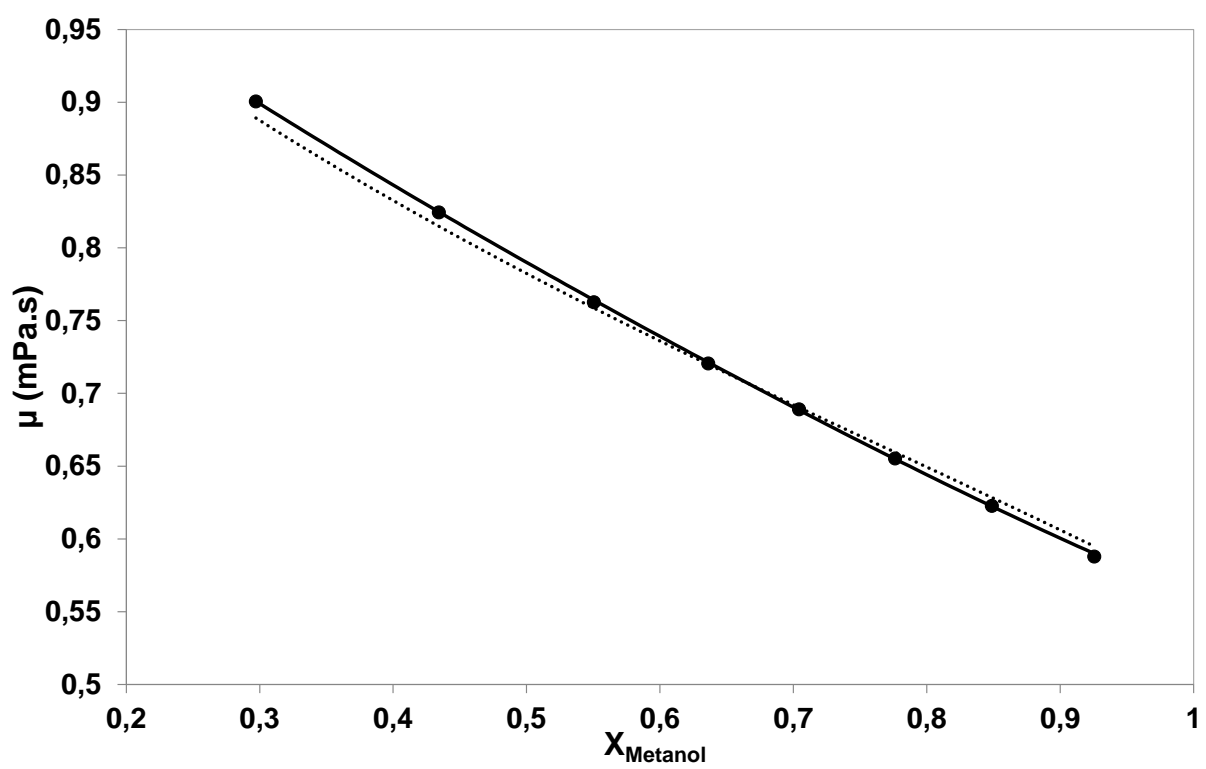

Figura 5 - viscosidade da mistura metanol + etanol. $k_{i j}, \sigma_{0}, \sigma_{1}(-)$ e $k_{i j}(\cdots) .(\bullet)$ (Wei e Rowley, 1984).

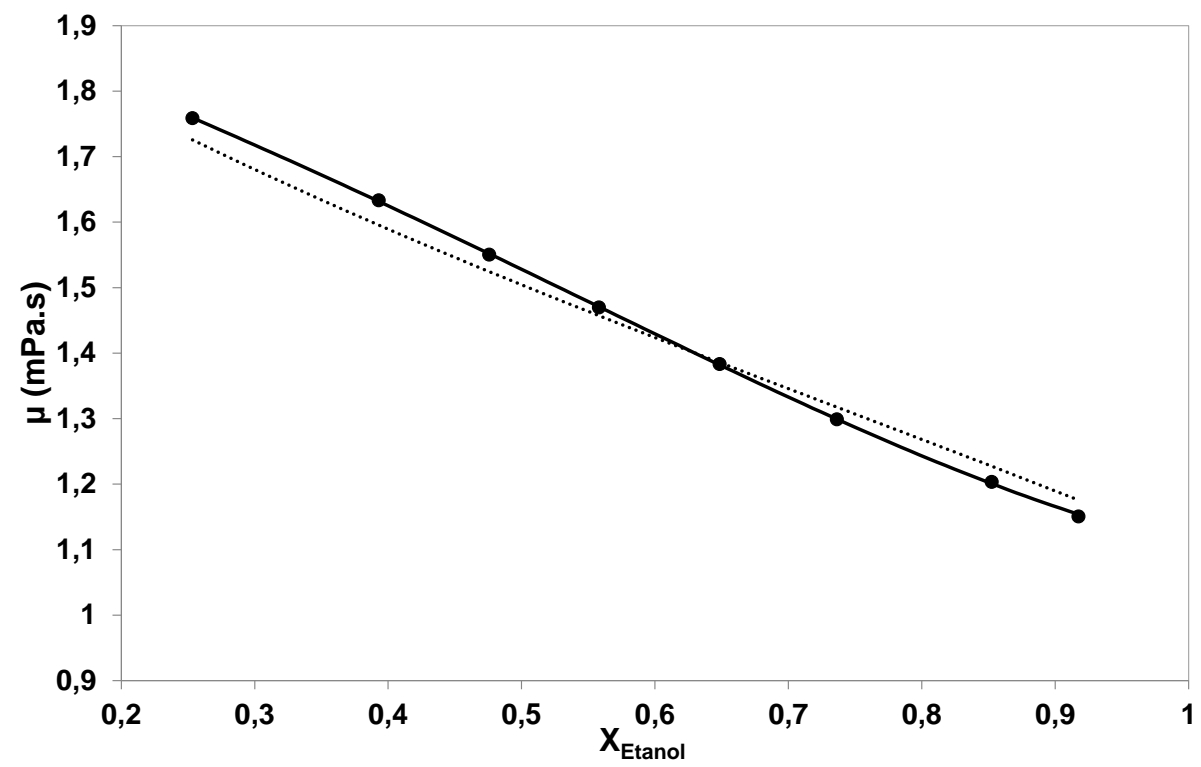

Figura 6 - Viscosidade da mistura Etanol +2- propanol. $k_{i j}, \sigma_{0}, \sigma_{1}(-)$ e $k_{i j}(\cdots)$. (•) (Wei e Rowley, 1984). 